\title{
Molecular Analysis and Antimicrobial Resistance Pattern of Tigecycline-Non-Susceptible K. pneumoniae Isolated from a Tertiary Care Hospital of East Asia
}

\author{
Niya $\mathrm{Hu}^{\mathrm{l}}$,* \\ Dongjiang Wang ${ }^{2, *}$ \\ Yiqing Lin' \\ Jun Zou ${ }^{3}$ \\ Yanling Liu' \\ Zhigang Xiong ${ }^{3}$ \\ Jian Guo ${ }^{2}$ \\ Lingbing Zeng' \\ Junming $\mathrm{Li} \mathbb{D}^{\prime}$
}

'Department of Clinical Laboratory, The First Affiliated Hospital of Nanchang University, Nanchang, People's Republic of China; ${ }^{2}$ Department of Laboratory Medicine, Shanghai East Hospital, Tongji University, School of Medicine, Shanghai, People's Republic of China; ${ }^{3}$ Department of Orthopedics, Jiangxi Provincial Children's Hospital, Nanchang, People's Republic of China

*These authors contributed equally to this work
Correspondence: Lingbing Zeng; Junming Li Email lingbing_zeng@163.com; lisir36I@I63.com
Introduction: Tigecycline is one of the last resorts for carbapenem-resistant $K$. pneumoniae (CRKP) infections. Indeed, tigecycline-non-susceptible $K$. pneumoniae (TNSKP) strains are increasingly treated with the use of tigecycline. In this study, we attempted to better understand their epidemiological trends and characteristics. K. pneumoniae were collected from 2017 to 2020 at the First Affiliated Hospital of Nanchang University.

Methods: Thirty-four TNSKP strains were selected during the study period, all of which were analyzed using antimicrobial susceptibility testing, multilocus sequence typing (MLST), and pulsed-field gel electrophoresis (PFGE). PCR and DNA sequencing were performed for the detection of $\beta$-lactamase genes and carbapenemase genes, and the mutation analysis of $\operatorname{tet}(A), \operatorname{tet}(X), \operatorname{tet}(L), \operatorname{tet}(M), \operatorname{rps} J, \operatorname{ram} R$, and $\operatorname{oq} x R$, which are related to tigecycline resistance. Virulence gene and capsular genotype testing were conducted to identify whether the TNSKP strains were hypervirulent Klebsiella pneumoniae.

Results: An epidemiology analysis showed that Klebsiella pneumoniae carbapenemase-2 $(K P C-2)$ was the predominant carbapenemase in tigecycline non-susceptible carbapenemresistant $K$. pneumoniae (TNSCRKP) (96.7\%), and the dominant clone type was ST11K14K64 (82.4\%). Among them, 55.9\% (19/34) of strains were from each department of ICU, particularly EICU and neurosurgery ICU. In order to further understand the molecular mechanisms of the TNSKP, a polymerase chain reaction of the resistant determinants was carried out. The results detected many tigecycline-resistant genes, such as $\operatorname{tet}(A)(97.1 \%)$, tet (X) (17.6\%), rpsJ (97.1\%), and $\operatorname{ramR}(8.8 \%)$.

Conclusion: As the results of this study reveal, we should take effective measures to control the increase in TNSKP.

Keywords: carbapenem-resistant, tigecycline-non-susceptible, Klebsiella pneumoniae

\section{Introduction}

Klebsiella pneumoniae belongs to the Enterobacteriaceae family and is an opportunistic pathogen that can transfer to multi-drug resistant strains and can cause various infections, including bacteremia, pneumonia, liver abscess, and urinary tract infection. ${ }^{1,2}$ At present, the spread of multi-drug resistant $K$. pneumoniae strains is a worldwide problem, and especially the carbapenem-resistant $K$. pneumoniae (CRKP); their infections are usually associated with high mortality. ${ }^{3}$ 
Tigecycline is a novel type of broad-spectrum glycyltetracycline antibiotics administered after minocycline development. It has a very wide range of activities against most Gram-positive bacteria, Gram-negative bacteria, and anaerobic bacteria, especially for multi-drug resistant strains. ${ }^{4,5}$ Tigecycline is one of the few available options for the treatment of carbapenem-resistant bacterial infections. ${ }^{6}$ Thus, tigecycline is considered to be the last line of defense for the treatment of this kind of bacterial infection. However, tigecycline-insensitive strains of K. pneumoniae are increasingly being reported, with some reports showing emergence in Taiwan, ${ }^{7}$ Northern China, ${ }^{8}$ Korea, ${ }^{9}$ Austria, ${ }^{10}$ etc. According to the China Antibiotic Resistance Surveillance System for antibiotic resistance, the tigecycline-non-susceptible K. pneumoniae (TNSKP) was up to $8.5 \%$ in 2019 (http:// www.carss.cn/); thus, we should pay more attention to them in clinical infections.

In order to explore whether tigecycline-non-susceptible K. pneumoniae are also present in the First Affiliated Hospital of Nanchang University, and then to better understand their epidemiological trends and characteristics, we analyzed the K. pneumoniae in the hospital from 2017 to 2020, and screened for tigecycline-non-susceptible K. pneumoniae in order to perform further analysis. We aimed to provide more advice that may be useful for controlling the infection rates in the hospital.

\section{Materials and Methods}

\section{Bacterial Strains and Data Collection}

In this study, we collected $5729 \mathrm{~K}$. pneumoniae nonduplicate strains from the First Affiliated Hospital of Nanchang University from 2017 to 2020. Cases of infection with tigecycline-non-susceptible $K$. pneumoniae strains were detected at different wards, and strains were isolated from different types of clinical specimens. Detailed clinical data were obtained from electronic medical records and informed consent was obtained from all patients included in the study. A concise flow chart is shown in Figure 1. All clinical isolates were identified using MALDI-TOF MS (Bruker, Sancordon Inc., Bremen, Germany). K. pneumoniae ATCC 13883 was used as a quality control strain for antimicrobial susceptibility testing. The Salmonella enterica serotype Braenderup strain (H9812) was used as a reference standard of pulsed-field gel electrophoresis (PFGE).

\section{Antimicrobial Susceptibility Testing}

The MICs of amoxicillin-clavulanic acid, piperacillintazobactam, ceftazidime, ceftriaxone, ertapenem, imipenem, meropenem, amikacin, ciprofloxacin, and tigecycline were determined using the broth microdilution methodology. Except for tigecycline, the sensitivity of other antibacterial agents was determined with reference to the CLSI M100 guidelines (2020). The interpretive criteria for tigecycline was according to US Food and Drug Administration (FDA) standards.

\section{Molecular Detection of Resistance Genes}

We carried out polymerase chain reaction detection of $\beta$ lactamase genes, including $b l a_{T E M}, b l a_{S H V}, b l a_{C T X-M}$, and $b l a_{L A P}$, and carbapenemase genes, including bla $a_{V I M}$,

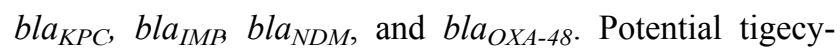
cline-resistance determinants containing $\operatorname{tet}(A), \operatorname{tet}(X)$, tet $(L), \operatorname{tet}(M), \operatorname{rps} J$, ramR, and $o q x R^{11-13}$ were also amplified in the tigecycline-non-susceptible strains and sequenced in positive strains; then, rpsJ, ramR, and oqxR were compared with the wild-type reference strain $K$. pneumoniae MGH78578 (GenBank accession number CP000647) as previously described. ${ }^{11}$ The primers used to amplify the coding sequence of each gene are listed in Table S1.

\section{Molecular Detection of Virulence Genes and Capsular Genotype}

It will be more difficult to treat clinically strains that express both virulence genes and are tigecycline nonsusceptible. Thus, we detected virulence genes, including rmpA, aero, uge, ironB, $m r k D$, and $k p n$, and we used the $w z i$ capsule gene to determine the capsule genotype (K-type) in the tigecycline non-susceptible strains.

\section{Multilocus Sequence Typing (MLST) and Pulsed-Field Gel Electrophoresis (PFGE) for Tigecycline-Non-Susceptible Strains}

According to the protocol described on the MLST Klebsiella pneumoniae website (https://bigsdb.pasteur.fr/klebsiella/pri mers used.html), all tigecycline non-susceptible strains were tested with seven housekeeping genes (rpoB, gapA, $m d h$, pgi, phoE, infB, and $\operatorname{ton} B^{14}$ ). The results of MLST were analyzed using the international Klebsiella pneumoniae MLST database established at the Pasteur Institute in France in 2005. ${ }^{14}$ Allele sequence and sequence type (ST) are verified on https://bigsdb.pasteur.fr/klebsiella/. PFGE was performed as previously described, ${ }^{15}$ the screened 
tigecycline non-susceptible $K$. pneumoniae strains were picked from fresh pure colonies into the buffer, and each of them was placed into small plastic containers. The DNA was digested by the restriction enzyme XbaI (TakaRa, Japan) $10 \mathrm{U}$ for $4 \mathrm{~h}$ at $37^{\circ} \mathrm{C}$. Electrophoresis was conducted using $0.5 \times \mathrm{TBE}$ buffer at $14^{\circ} \mathrm{C}$ for $18 \mathrm{~h}$, and pulse time was 2-64 s. Salmonella enterica serotype Braenderup strain (H9812) was used as a classical molecular weight standard. We finally used Bionumerics software (Applied Maths, SintMartens-Latem, Belgium) to analyze homogeneity. Clinical isolates with more than $80 \%$ homology were defined as the same PFGE cluster.

\section{Results}

\section{Clinical Characteristics of Patients Infected with TNSKP}

In this study, a total of $5729 \mathrm{~K}$. pneumoniae strains were collected, there were 1282, 1397, 1564, and 1486 strains in 2017-2020, respectively, and of them 1851 (32.3\%, 1851/ 5729) strains were CRKP. The $K$. pneumoniae strains showed high susceptibility to TGC, but we also found 34 patients infected with TNSKP; the demographic and clinical characteristics of the 34 strains are shown in Figure 2. Among the 34 isolates, the number of specimens from sputum was the largest, accounting for $64.7 \%$ (22/34), and the number of specimens from urine $17.6 \%(6 / 34)$ ranked second, followed by blood 5.9\% (2/34), ascites $5.9 \%(2 / 34)$, catheter tip $2.9 \%(1 / 34)$, and wound fluid $2.9 \%(1 / 34)$. The TNSKP strain was not found in 2017 , but the TNSKP rates rose during the following years, by $0.1 \%$

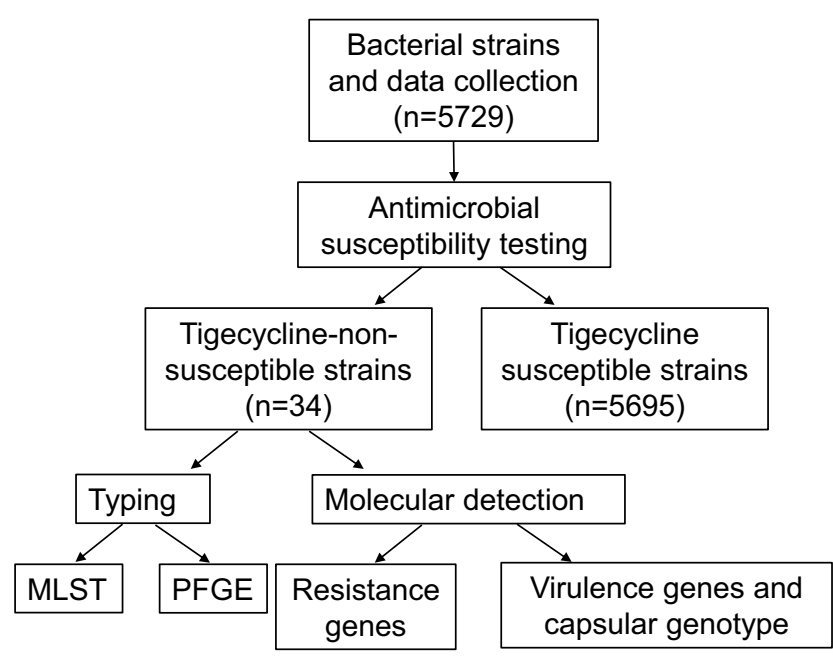

Figure I A concise flow chart about this study.
(2/1397), $0.6 \%(9 / 1564)$, and $1.5 \%$ (23/1486) in 2018-2020, respectively. Analysis of the departmental distribution of the patients revealed that $55.9 \%(19 / 34)$ of the patients with nonsusceptible strains were from the intensive care unit (ICU), particularly from EICU $14.7 \%$ (5/34) and neurosurgery ICU $14.7 \%(5 / 34)$. In 2018, both of the TNSKP strains were from neurosurgery ICU. In addition to this, common departments also had more TNSKP strains, such as the rehabilitation department $11.8 \%(4 / 34)$ and neurosurgery $11.8 \%(4 / 34)$.

\section{Antibiograms}

The antibiotic resistance results of TNSKP strains to 10 antibiotics are shown in Table 1. We can see that 34 strains showed low susceptibility to all of the 10 antibiotics, including piperacillin/tazobactam (11.8\%), ceftazidime (2.9\%), ceftriaxone (2.9\%), ertapenem (11.8\%), imipenem (14.7\%), meropenem (14.7\%), amikacin (17.6\%), and ciprofloxacin (5.9\%); acid susceptibility rates to amoxicillin/clavulanic were zero. Among the 34 tested TNSKP strains, 30 (30/34, $88.2 \%$ ) were also CRKP strains. According to the interpretive criteria for tigecycline ( $\leq 2 \mu \mathrm{g} / \mathrm{mL}$, susceptible; $4 \mu \mathrm{g} / \mathrm{mL}$, intermediate; $\geq 8 \mu \mathrm{g} / \mathrm{mL}$, resistant), there were three $(3 / 34$, $8.8 \%$ ) intermediate strains; the others were resistant.

\section{Analysis of Resistance Determinants in TNSKP Strains}

To investigate the resistance mechanism of TNSKP, potential tigecycline resistance determinants were identified in 34 strains by PCR and sequencing. The genes $\operatorname{tet}(A)$ and rps $J$ were detected in most of the TNSKP strains except for strain No. 44; $\operatorname{tet}(X)$ was found in six strains (17.6\%), whereas $\operatorname{tet}(L)$ and $\operatorname{tet}(M)$ were not detected in all of the TNSKP strains. Three strains $(8.8 \%)$ were detected with ramR expression; similarly, oqxR was found in three $(8.8 \%)$ isolates. One strain with S29L substitutions in ramR and another two strains with a termination codon appeared in advance. Meanwhile, mutations in rps $J$ and oqxR were not identified in this study (Table 2).

A total of five carbapenemases were detected in this study; $96.7 \%$ (29/30) of Tigecycline non-susceptible carbapenem-resistant $K$. pneumoniae (TNSCRKP) were bla $_{k p c}$ positive (Table 2); and bla ${ }_{V I M}$, bla $_{I M B}$ bla $_{N D M}$, and bla $a_{O X A-48}$ were not detected in any of the strains. Among the 34 strains, it was detected that 29 strains carried $b l a_{S H V-12}, 2$ strains carried both $b l a_{S H V-158}$ and $b l a_{S H V-187}$, 30 strains carried $b_{T E M-1}$, and 1 strain carried bla $_{T E M-235}$. A total of 31 strains carried bla ${ }_{C T X-M-65}, 1$ strain carried 
both bla $_{C T X-M-3}$ and $b l a_{C T X-M-14}$, and 31 strains carried $b l a_{L A P-2}$. For strain No. 11 only, no carbapenemases were detected while in CRKP.

\section{Virulence Analysis}

All strains in the study carried the $m r k D$ gene, followed by kpn $(97.1 \%, 33 / 34)$ and uge $(91.2 \%, 31 / 34)$. Only one strain carried the rmpA gene, while aero and iroN were not detected among any of the strains. Two or more genes coexisted in these isolates; the combination of uge, $m r k D$, and kpn was the most common prototype observed, and there were 29 isolates of this type. Three isolates were a combination of $m r k D$ and $k p n$, while one combination of $m r k D$ and uge was found. There was only one strain, No. 25, that was a combination of rmpA, uge, $m r k D$, and kpn (Table 2).

\section{Isolates Typing and PFGE}

In total, five different multilocus sequence types were detected; ST11 was the most prevalent type $(85.3 \%, 29 /$ $34)$, followed by ST37 (5.9\%, 2/34), and only one strain each of ST3627, ST2593, and ST2601 (Figure 2).

The wzi capsular gene was sequenced to assign a capsular type (K-type) to each isolate. In this study, two K-types were identified. A total of $88.2 \%(30 / 34)$ of the isolates were typed as K14.K64, while 8.8\% (3/34) were typed as K15.K17.K50.K51.K52. One strain, No. 63, was not linked to any K-type (Figure 2).
According to the results of PFGE, the similarity of the strains ranged between $60 \%$ and $100 \%$. Among them, the 34 strains were divided into six different PFGE clusters (clusters A to F) at an $82 \%$ similarity cut-off (Figure 2). Obviously, the dominant strain species was cluster A, which contained a total of 28 strains $(82.4 \%, 28 / 34)$, followed by cluster D $(5.9 \%, 2 / 34)$; each of the remaining four cluster (B/C/E/F) contained only one strain; 29 ST11 isolates mainly belonged to cluster A.

\section{Discussion}

The prevalence of carbapenem-resistant K. pneumoniae has been increasing globally, making antimicrobial treatment difficult and leading to higher disease-related mortality. ${ }^{16,17}$ In this study, we collected 5729 K. pneumoniae strains from our hospital over four years; carbapenem resistance rates in $K$. pneumoniae reached $32.3 \%$, which is higher than the average level in Jiangxi province according to the China Antibiotic Resistance Surveillance System in 2019 (http://www.carss.cn/). This may be related to the large number of severe patients in our hospital, and because of long hospital stays, most of the TNSKP strains were from the intensive care unit. With the increasing administration of tigecycline in clinical settings, tigecycline-non-susceptible strains will become increasingly prominent. The TNSKP rates were below $8.5 \%$ at present in our hospital, but we should pay more attention to this issue, in light of the rising trend detected over our four-year study period. Meanwhile, we found that

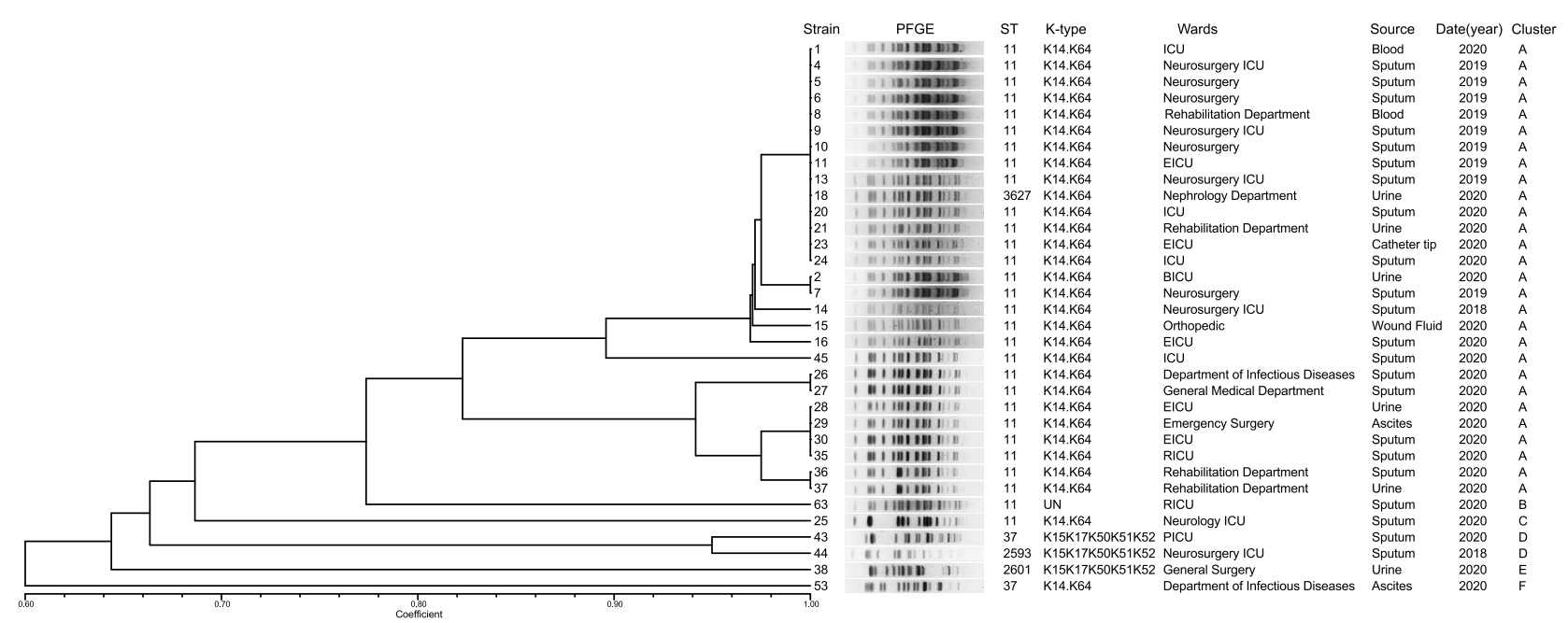

Figure 2 PFGE results for 34 TNSKP strains. The adjacent information shown on the right represents ST, K-type of the sequenced isolates, wards, source, and cluster in turn.

Abbreviations: PFGE, pulsed-field gel electrophoresis; UN, unknown; ICU, intensive care unit; EICU, emergency intensive care unit; BICU, burn intensive care unit; RICU, respiratory intensive care unit; PICU, pediatric intensive care unit. 


\begin{tabular}{|c|c|c|}
\hline & 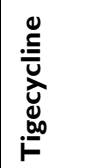 & 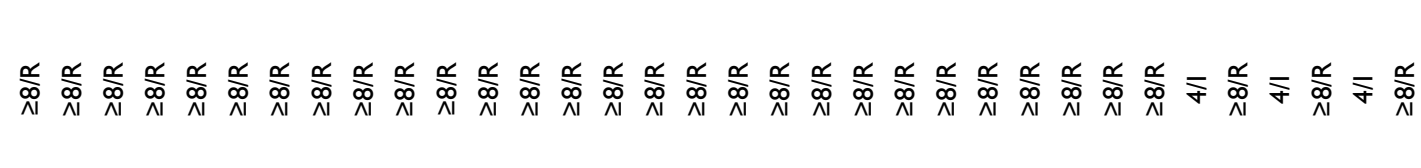 \\
\hline & 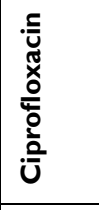 & 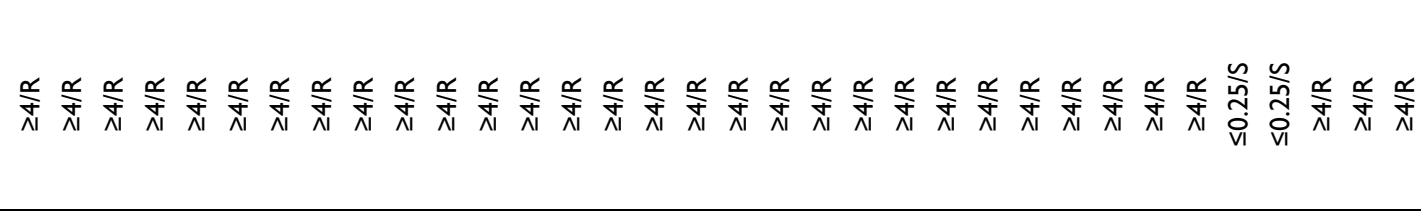 \\
\hline & 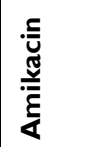 & 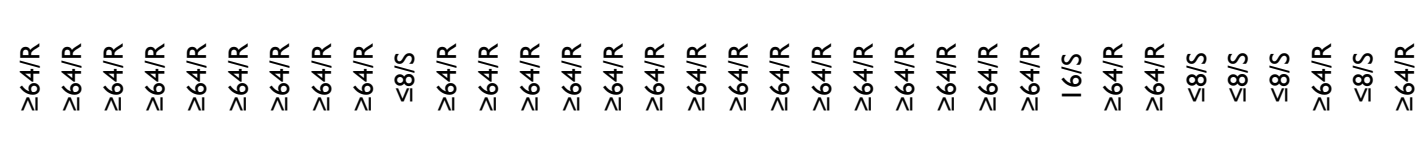 \\
\hline & 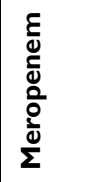 & 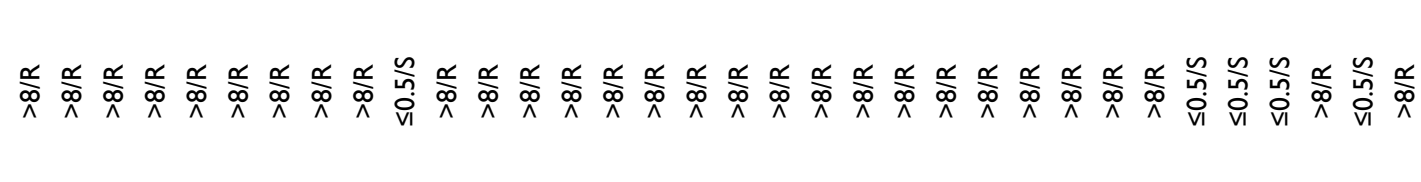 \\
\hline & 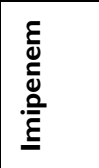 & 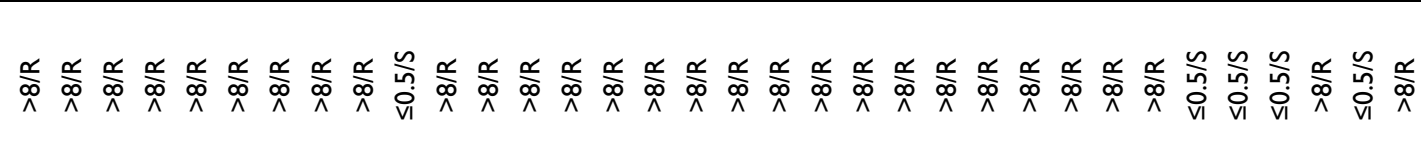 \\
\hline 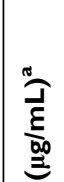 & 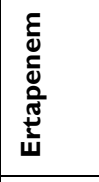 & 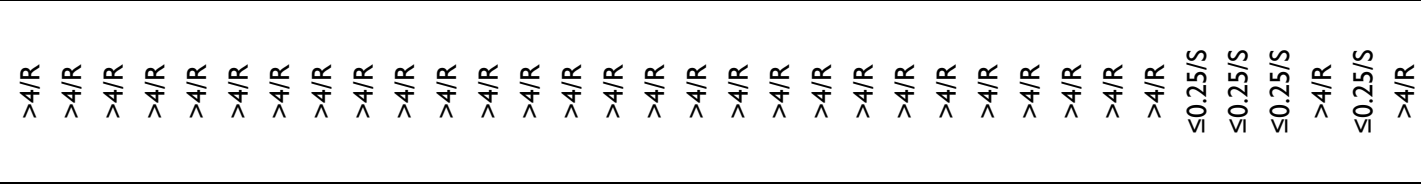 \\
\hline$\frac{u}{\Sigma}$ & 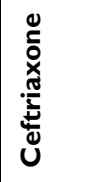 & 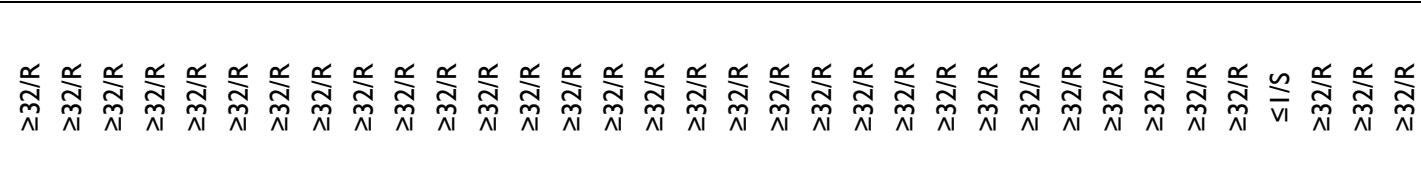 \\
\hline & 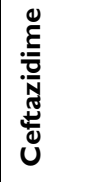 & 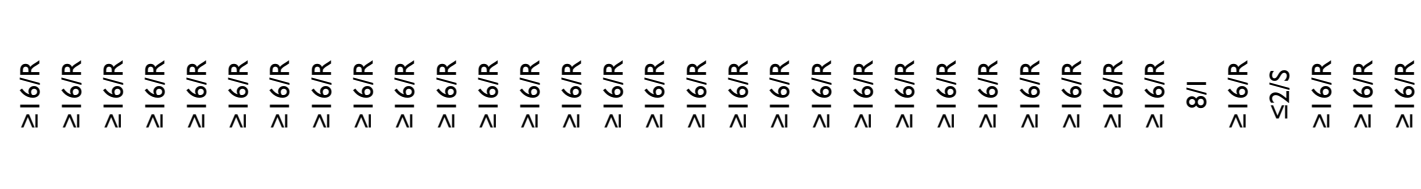 \\
\hline & 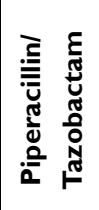 & 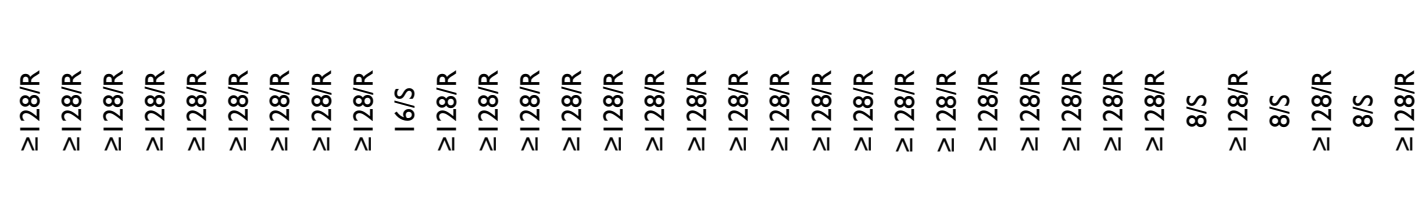 \\
\hline & 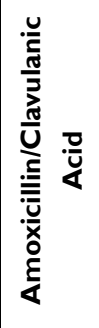 & 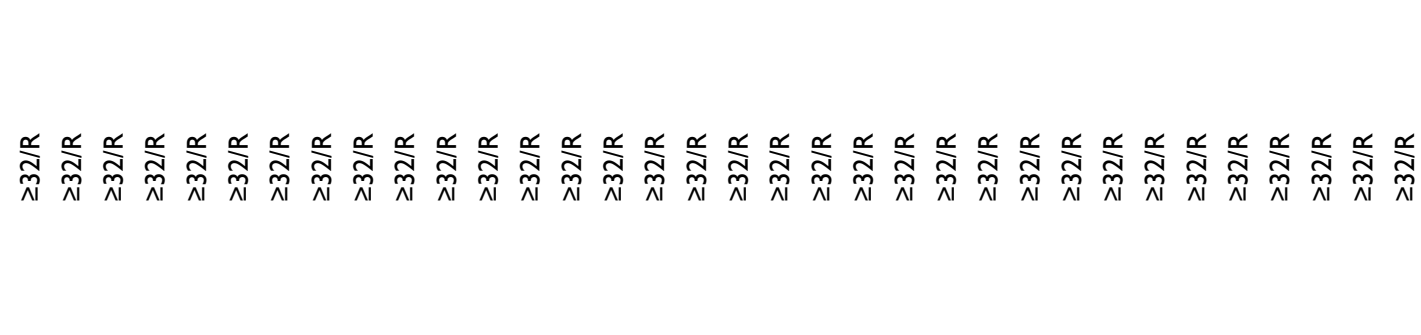 \\
\hline & & ఇ \\
\hline
\end{tabular}




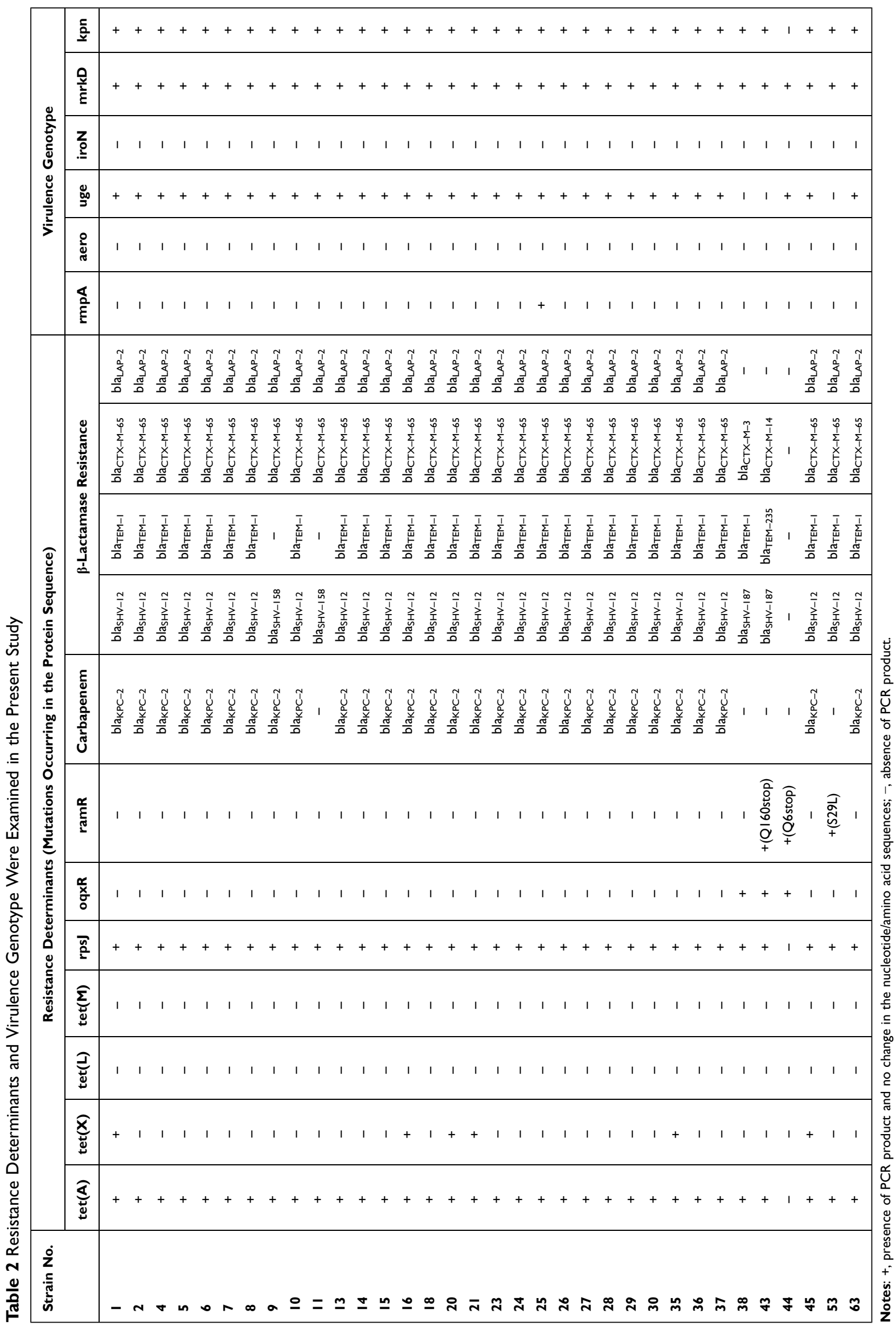


most of the TNSKP strains were also resistant to carbapenems, which is consistent with other studies; ${ }^{18}$ hence, the antimicrobial susceptibility testing confirmed low susceptibility to all of the 10 antibiotics. According to the antibiotic resistance results of tigecycline, there were three intermediate strains (strains No. 38, 44, and 53), and among them two strains with mutations in $\operatorname{ram} R$ (strains No. 44 and 53), and two strains were detected with oqxR expression (strains No. 38 and 44). To our knowledge, the AcrAB-TolC efflux pump associated with the inactivation of the repressor ram $R$ caused by mutations is a major cause of tigecycline resistance. ${ }^{19}$ In addition to this, the OqxAB efflux pump is downregulated by the repressor oqxR, which was also associated with tigecycline resistance. ${ }^{20}$ Thus, our results are in accordance with the above. Except for efflux-mediated resistance mechanisms, there were other resistance mechanisms such as Tet proteins, including mutated $\operatorname{tet}(A), \operatorname{tet}(L), \operatorname{tet}(X)$, and tet $(M)$, and the rps $J$ gene, which was associated with an alteration in the tigecycline target site of the ribosomal protein S10. ${ }^{12}$ In this study, $31 / 34(91.2 \%)$ strains were tigecycline-resistant, and all of them were detected as $\operatorname{tet}(A)$ and rps $J$ gene-positive, although mutations were not identified. We suspect that they may be the main cause of the resistance mechanisms among these strains, but further studies are needed.

At present, common carbapenemase genes, including bla $a_{V I M}, b l a_{K P C}, b l a_{I M B} b l a_{N D M}$, and bla $a_{O X A-48}$, as well as $K P C$-type enzymes, and in particular $K P C$-2, are the most common carbapenemases in China. ${ }^{21,22}$ As our data show, 96.7\% (29/30) of Tigecycline non-susceptible carbapenem-resistant $K$. pneumoniae were detected with bla $_{K P C-2}$, which is consistent with previous reports. Only for strain No. 11 was no carbapenemase detected in this study. Strain No. 11 was ertapenem-resistant but imipenem- and meropenem-susceptible, and showed $b^{b} a_{S H V-158}, \quad b l a_{C T X-M-65}$, and $b l a_{L A P-2}$ beta-lactamases resistance; previous studies showed that the mechanisms of resistance in this form would be predominantly mediated by non-carbapenemase mechanisms, including deficiencies in the expression of porins Omp35 and Omp $36^{23}$ or the production of particular betalactamases. $^{24}$

Among the TNSKP isolates, we detected the virulencerelated genes $m r k D, k p n$, and $u g e$ with a positive rate more than $90 \%$; these genes are associated with adhesins and lipopolysaccharides of $K$. pneumoniae. ${ }^{25}$ Only one strain was positive for the $\operatorname{rmp} A$ gene, which is related to the high viscosity of $K$. pneumoniae; ${ }^{26}$ aero and iroN, which are associated with iron carriers, were not detected in this study. In addition, in this experiment we determined capsular types by sequencing the wzi gene, and they were mainly the K14.K64 type, whereas previous studies reported the K47 type as dominant. ${ }^{27}$ In this study, we did not find virulent capsular types, such as K1, K2, K5, $\mathrm{K} 16$, K20, K54, and K57, and thus these TNSKP isolates carried high resistance but low virulence.

Molecular typing of our strains showed the dominant sequence type of the TNSKP isolates to be ST11, which is in accordance with reports that ST11 is the most common type of CRKP associated with the spread of bla $_{\mathrm{KPC}-2}$ in China. ${ }^{28,29}$ Other STs, such as ST37, ST3627, ST2593, and ST2601, were also detected in 34 TNSKP strains, which was different from previous reports in Henan. ${ }^{30}$ Our study is the first to report of ST3627, ST2593, and ST2601 K. pneumoniae with tigecycline-non-susceptible caused by clinical infection. PFGE results showed that the most prevalent clone type ST11-K14K64 (28/34, 82.4\%) belongs to cluster A. In cluster A, the ST11-K14K64 isolates were mainly found in the ICU, neurosurgery ICU, and EICU, which indicated that there may have been a prevalence of ST11K14K64 TNSKP in the ICU of our hospital from 2018 to 2020.

\section{Conclusion}

The rate of carbapenem-resistant $K$. pneumoniae in our hospital was higher than the average level in Jiangxi province, the tigecycline-non-susceptible $K$. pneumoniae was often found in carbapenem-resistant strains, and they were usually isolated from the intensive care unit. The ST11-K14K64 clone type carrying the $b l a_{K P C-2}$ was primarily detected in this study, and the $\operatorname{tet}(A)$ and rps $J$ genes may contribute to the tigecycline-non-susceptible phenotype in the 34 TNSKP strains, which is worthy of further research. In all, we should take effective measures, such as active surveillance and effective segregation to prevent CRKP, thus reducing the emergence of TNSKP.

\section{Abbreviations}

CRKP, carbapenem-resistant $K$. pneumoniae; TNSKP, tigecycline-non-susceptible $K$. pneumoniae; TNSCRKP, tigecycline non-susceptible carbapenem-resistant K. pneumoniae; PFGE, pulsed-field gel electrophoresis; CLSI, the Clinical and Laboratory Standards Institute; FDA, Food and Drug Administration; MLST, multilocus 
sequence typing; ST, sequence type; ICU, intensive care unit.

\section{Data Sharing Statement}

The data used and/or analyzed in this study are available from the Lingbing Zeng (E-mail: lingbing_zeng@163. com) and Junming Li (E-mail: lisir361@163.com) on reasonable request.

\section{Ethics Approval}

This study was approved by the Ethics Committee of the first affiliated hospital of Nanchang University (Code: 2014056). All participants in this study provided written informed consent. This study was conducted in accordance with the Declaration of Helsinki.

\section{Author Contributions}

All authors made a significant contribution to the work reported, whether that is in the conception, study design, execution, acquisition of data, analysis and interpretation, or in all these areas; took part in drafting, revising or critically reviewing the article; gave final approval of the version to be published; have agreed on the journal to which the article has been submitted; and agree to be accountable for all aspects of the work.

\section{Funding}

This work was supported by the National Natural Science Foundation of China (32060040 and 31760261), the National Science Foundation of Jiangxi province (20202BABL216084 and 20192ACBL21042), and the Science and Technology Plan Projects of Health and Family Planning Commission of Jiangxi province (20203692).

\section{Disclosure}

The authors declare no conflicts of interest.

\section{References}

1. Ko WC, Paterson DL, Sagnimeni AJ, et al. Community-acquired Klebsiella pneumoniae bacteremia: global differences in clinical patterns. Emerg Infect Dis. 2002;8:160-166. doi:10.3201/ eid0802.010025

2. Siu LK, Yeh KM, Lin JC, Fung CP, Chang FY. Klebsiella pneumoniae liver abscess: a new invasive syndrome. Lancet Infect Dis. 2012;12:881-887. doi:10.1016/S1473-3099(12)70205-0

3. Munoz-Price LS, Poirel L, Bonomo RA, et al. Clinical epidemiology of the global expansion of Klebsiella pneumoniae carbapenemases. Lancet Infect Dis. 2013;13:785-796. doi:10.1016/S1473-3099(13) 70190-7
4. Slover CM, Rodvold KA, Danziger LH. Tigecycline: a novel broad-spectrum antimicrobial. Ann Pharmacother. 2007;41:965-972. doi:10.1345/aph.1H543

5. Zhong X, Xu H, Chen D, Zhou H, Hu X, Cheng G. First emergence of acrAB and oqxAB mediated tigecycline resistance in clinical isolates of Klebsiella pneumoniae pre-dating the use of tigecycline in a Chinese hospital. PLoS One. 2014;9:e115185. doi:10.1371/journal.pone. 0115185

6. Lee CR, Lee JH, Park KS, Kim YB, Jeong BC, Lee SH. Global dissemination of carbapenemase-producing Klebsiella pneumoniae: epidemiology, genetic context, treatment options, and detection methods. Front Microbiol. 2016;7:895. doi:10.3389/ fmicb.2016.00895

7. Cheng YH, Huang TW, Juan CH, et al. Tigecycline-non-susceptible hypervirulent Klebsiella pneumoniae strains in Taiwan. J Antimicrob Chemother. 2020;75:309-317. doi:10.1093/jac/dkz450

8. Xu H, Zhou Y, Zhai X, et al. Emergence and characterization of tigecycline resistance in multidrug-resistant Klebsiella pneumoniae isolates from blood samples of patients in intensive care units in northern China. J Med Microbiol. 2016;65:751-759. doi:10.1099/ jmm.0.000299

9. Jeong SH, Kim HS, Kim JS, et al. Prevalence and molecular characteristics of carbapenemase-producing Enterobacteriaceae from five hospitals in Korea. Ann Lab Med. 2016;36:529-535. doi:10.3343/ alm.2016.36.6.529

10. Hladicz A, Kittinger C, Zarfel G. Tigecycline resistant Klebsiella pneumoniae isolated from Austrian River Water. Int J Environ Res Public Health. 2017;14:1169. doi:10.3390/ijerph14101169

11. Chiu SK, Huang LY, Chen H, et al. Roles of $\operatorname{ramR}$ and tet(A) mutations in conferring tigecycline resistance in carbapenem-resistant Klebsiella pneumoniae clinical isolates. Antimicrob Agents Chemother. 2017;61. doi:10.1128/AAC.00391-17

12. Villa L, Feudi C, Fortini D, Garcia-Fernandez A, Carattoli A. Genomics of KPC-producing Klebsiella pneumoniae sequence type 512 clone highlights the role of RamR and ribosomal S10 protein mutations in conferring tigecycline resistance. Antimicrob Agents Chemother. 2014;58:1707-1712. doi:10.1128/AAC.01803-13

13. Moore IF, Hughes DW, Wright GD. Tigecycline is modified by the flavin-dependent monooxygenase TetX. Biochemistry. 2005;44:11829-11835. doi:10.1021/bi0506066

14. Diancourt L, Passet V, Verhoef J, Grimont PA, Brisse S. Multilocus sequence typing of Klebsiella pneumoniae nosocomial isolates. J Clin Microbiol. 2005;43:4178-4182. doi:10.1128/JCM.43.8.41784182.2005

15. Gona F, Comandatore F, Battaglia S, et al. Comparison of core-genome MLST, coreSNP and PFGE methods for Klebsiella pneumoniae cluster analysis. Microb Genom. 2020;6. doi:10.1099/ mgen.0.000347

16. Nordmann P, Cuzon G, Naas T. The real threat of Klebsiella pneumoniae carbapenemase-producing bacteria. Lancet Infect Dis. 2009;9:228-236. doi:10.1016/S1473-3099(09)70054-4

17. Neuner EA, Yeh JY, Hall GS, et al. Treatment and outcomes in carbapenem-resistant Klebsiella pneumoniae bloodstream infections. Diagn Microbiol Infect Dis. 2011;69:357-362. doi:10.1016/j. diagmicrobio.2010.10.013

18. Pournaras S, Koumaki V, Spanakis N, Gennimata V, Tsakris A. Current perspectives on tigecycline resistance in Enterobacteriaceae: susceptibility testing issues and mechanisms of resistance. Int $J$ Antimicrob Agents. 2016;48:11-18. doi:10.1016/j. ijantimicag.2016.04.017

19. Wang X, Chen H, Zhang Y, et al. Genetic characterisation of clinical Klebsiella pneumoniae isolates with reduced susceptibility to tigecycline: role of the global regulator RamA and its local repressor RamR. Int J Antimicrob Agents. 2015;45:635-640. doi:10.1016/j. ijantimicag.2014.12.022 
20. Veleba M, Higgins PG, Gonzalez G, Seifert H, Schneiders T. Characterization of RarA, a novel AraC family multidrug resistance regulator in Klebsiella pneumoniae. Antimicrob Agents Chemother. 2012;56:4450-4458. doi:10.1128/AAC.00456-12

21. Yu X, Zhang W, Zhao Z, et al. Molecular characterization of carbapenem-resistant Klebsiella pneumoniae isolates with focus on antimicrobial resistance. BMC Genomics. 2019;20:822. doi:10.1186/ s12864-019-6225-9

22. Zeng L, Deng Q, Zeng T, Liu Y, Zhang J, Cao X. Prevalence of carbapenem-resistant Klebsiella pneumoniae infection in Southern China: clinical characteristics, antimicrobial resistance, virulence, and geographic distribution. Microb Drug Resist. 2020;26:483-491. doi:10.1089/mdr.2018.0401

23. Chung HS, Yong D, Lee M. Mechanisms of ertapenem resistance in Enterobacteriaceae isolates in a tertiary university hospital. J Investig Med. 2016;64:1042-1049. doi:10.1136/jim-2016-000117

24. Wu JJ, Wang LR, Liu YF, Chen HM, Yan JJ. Prevalence and characteristics of ertapenem-resistant Klebsiella pneumoniae isolates in a Taiwanese university hospital. Microb Drug Resist. 2011;17:259-266. doi:10.1089/mdr.2010.0115

25. Candan ED, Aksoz N. Klebsiella pneumoniae: characteristics of carbapenem resistance and virulence factors. Acta Biochim Pol. 2015;62:867-874. doi:10.18388/abp.2015_1148
26. Yu WL, Ko WC, Cheng KC, et al. Association between rmpA and magA genes and clinical syndromes caused by Klebsiella pneumoniae in Taiwan. Clin Infect Dis. 2006;42:1351-1358. doi:10.1086/ 503420

27. Liu Y, Liu PP, Wang LH, Wei DD, Wan LG, Zhang W. Capsular polysaccharide types and virulence-related traits of epidemic KPC-producing Klebsiella pneumoniae isolates in a Chinese University Hospital. Microb Drug Resist. 2017;23:901-907. doi:10.1089/mdr.2016.0222

28. Qi Y, Wei Z, Ji S, Du X, Shen P, Yu Y. ST11, the dominant clone of KPC-producing Klebsiella pneumoniae in China. $J$ Antimicrob Chemother. 2011;66:307-312. doi:10.1093/jac/dkq431

29. Liu L, Feng Y, Tang G, et al. Carbapenem-resistant isolates of the Klebsiella pneumoniae complex in Western China: the common ST11 and the surprising hospital-specific types. Clin Infect Dis. 2018;67: S263-S265. doi:10.1093/cid/ciy662

30. Yan WJ, Jing N, Wang SM, et al. Molecular characterization of carbapenem-resistant Enterobacteriaceae and emergence of tigecycline non-susceptible strains in the Henan province in China: a multicenter study. J Med Microbiol. 2021;70. doi:10.1099/ jmm.0.001325
Infection and Drug Resistance

\section{Publish your work in this journal}

Infection and Drug Resistance is an international, peer-reviewed openaccess journal that focuses on the optimal treatment of infection (bacterial, fungal and viral) and the development and institution of preventive strategies to minimize the development and spread of resistance. The journal is specifically concerned with the epidemiology of
Dovepress

antibiotic resistance and the mechanisms of resistance development and diffusion in both hospitals and the community. The manuscript management system is completely online and includes a very quick and fair peerreview system, which is all easy to use. Visit http://www.dovepress.com/ testimonials.php to read real quotes from published authors. 